\section{Scleral penetration force requirements for commonly used intravitreal needles}

\begin{abstract}
Introduction Repeated intraocular injections have become routine practice. It is important to determine how to make them as painless as possible. We wished to examine the force required to penetrate the sclera with different gauge needles.

Methods Recently enucleated eyes had $4 \mathrm{~mm}$ scleral punch biopsies performed. The scleral disks were mounted on a fixed load cell and either a new needle or a needle that had previously penetrated a rubber vial was attached to a linear stage and the maximum penetration force measured.

Results The 27-gauge needles required

almost twice as much force to penetrate the sclera than either the 30 - or the 31 -gauge. In addition, the 30- and 31-gauge appeared to require similar force. In all cases, following vial perforation, there was an increase in the amount of force required to penetrate the sclera. The amount of force was variable Discussion Smaller gauge needles require less force to penetrate the sclera and needles that have been used to penetrate rubber vials require more force.

Eye (2007) 21, 1210-1211; doi:10.1038/sj.eye.6702577;

published online 1 September 2006
\end{abstract}

${ }^{1}$ Department of

Ophthalmology, Mayo

Clinic, Rochester, MN, USA

${ }^{2}$ Department of Orthopedic Biomechanics, Mayo Clinic, Rochester, MN, USA

Correspondence: JS Pulido, Professor of Ophthalmology, Department of Ophthalmology, Ocular Oncology and Vitreoretinal Diseases,

Mayo Clinic College of

Medicine,

Mayo Clinic,

200 First Street, SW,

Rochester, MN 55905, USA

Tel: 507-284-3721;

Fax: 507-284-4612.

E-mail: pulido.jose@

mayo.edu

Received: 30 June 2006

Accepted: 28 July 2006

Published online:

1 September 2006

Supported in part by an unrestricted grant from Research to Prevent Blindness Inc, NY
Keywords: intraocular injection; 30 gauge needles; scleral penetration

\section{Introduction}

Repeated intravitreal injections are now commonly performed for a variety of retinal diseases. The most commonly used needles for these injections are 27-, 30-, 31-gauges. ${ }^{1}$ The force that is required to penetrate the sclera using these various gauges is not known. We used a low-force testing system to determine the force to penetrate the sclera.
JS Pulido', ME Zobitz ${ }^{2}$ and K-N An²

Materials and methods

Recently enucleated eyes that had been fixed in formalin for 2 days were used. Using a 4-mm punch, peripheral circular scleral specimens were obtained. There were two punches performed per oblique quadrant avoiding the horizontal and vertical muscle insertions.

The disks were removed anterior to the equator. A new needle (Becton-Dickinson) was used as well as a needle that had previously perforated through the rubber top of a saline vial. The size of the needle was alternated with each disk to decrease the chances that a difference was only because of the variability between eyes. The maximum penetration force was measured using a custom-built, low-force micro-testing system. The test system consisted of a linear stage (Parker MX80L) mounted on an inverted microscope (Olympus CKX 41). The needle was connected to the linear stage and the specimen was attached to a fixed load cell. The needle was advanced at a rate of $0.25 \mathrm{~mm} / \mathrm{s}$ until it penetrated the specimen (Figure 1).

\section{Results}

A total of 12 different determinations were made - two with new 27-gauge needles, two with 30-gauge, and three with a new 31-gauge needle. The other five determinations were carried out following vial perforation - two with 27-gauge needles, one with a 30-gauge needle, and two with 31-gauge needles.

The 27-gauge needles required almost twice as much force to penetrate the sclera than either the 30 - or the 31-gauge (Figure 2). In addition, the 30- and 31-gauge appeared to require similar force.

In all cases, following vial perforation, there was an increase in the amount of force required to penetrate the sclera. The amount of force was variable (Figure 3) 


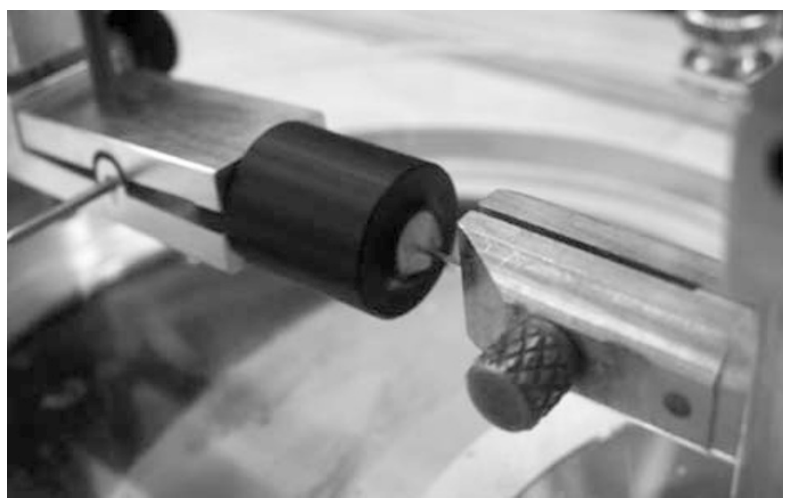

Figure 1 Instrumentation showing the 4-mm scleral disk on the fixed-load cell being perforated by the needle that is connected to the linear stage.

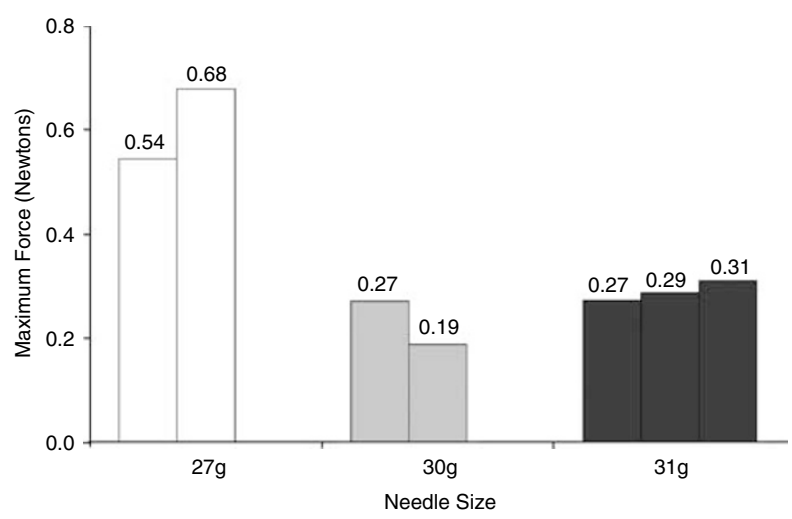

Figure 2 Bar graph showing the maximum force required to penetrate the sclera for various gauges of new needles.

\section{Discussion}

The maximum force required to penetrate the sclera is greater for the 27-gauge than for the 30- and 31-gauge

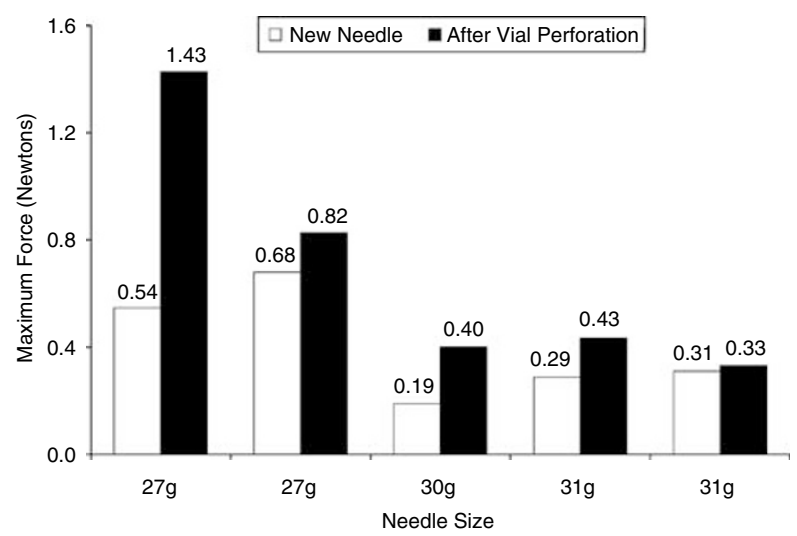

Figure 3 Bar graph comparing the maximum force required to penetrate the sclera for various gauges of new needles and needles that have previously gone through a rubber vial top.

needles. In addition, following vial perforation, the force required is increased for any gauge. Considering the present need for frequent intraocular injections in some patients, the need for making the injections as painless as possible is needed. Some of the determinants are patient-based that is, the thickness and calcification of the sclera. Calcification is more commonly seen near the insertion of the rectus muscles, and this area should be avoided during injections. Regarding the needles, the thinner the needle, the less surface area that is penetrated, therefore being less chance of touching nerve fibres. In addition, it appears that thinner needles require less force to penetrate the sclera than the larger ones.

\section{Reference}

1 Pulido JS, Pulido CM, Bakri SJ, McCannel CA, Cameron JD. The use of 31-gauge needles for recurrent intraocular injections. Eye 2006 Accepted. 